\title{
Ventilación pulmonar protectora, poder mecánico y presión de distensión pulmonar relacionado con mortalidad y SOFA en ventilación mecánica invasiva
}

Lung protective ventilation, mechanical power, driving pressure and its relation to mortality and SOFA in patients with invasive mechanical ventilation Ventilação pulmonar protetora, potência mecânica e pressão distensão pulmonar e sua relação com mortalidade e SOFA em pacientes com ventilação mecânica invasiva

José Iván Rodríguez de Molina Serrano, ${ }^{*}$ Hugo Humberto Castro Martínez, ${ }^{*}$ Ana Karen Oyarvide Zapuche, ${ }^{*}$ Gustavo Adolfo Flores Orta*

\section{RESUMEN}

Introducción: La ventilación mecánica es la piedra angular del tratamiento de los pacientes con insuficiencia respiratoria aguda; sin embargo, no está exenta de complicaciones y eventos adversos.

Objetivo: Describir la relación entre la EVPP, PM y la PDP con mortalidad y SOFA en pacientes adultos con VMI atendidos en el Hospital General de Zona No. 11, IMSS, Piedras Negras, Coahuila.

Material y métodos: Estudio prospectivo, observacional, de septiembre 2019 a marzo 2020 de pacientes adultos con ventilación mecánica invasiva. La variable independiente fue EVPP, PM y la PDP. Las dependientes fueron la mortalidad y el SOFA.

Resultados: Ingresaron 63 pacientes. Características principales: edad 55 años, sexo femenino $44.4 \%$, ubicación: piso $50.8 \%$, urgencias $17.5 \%$ y UTI $31.8 \%$. Diagnósticos: sepsis $20.6 \%$, TCE severo $7.8 \%$, EVC $7.8 \%$, NAC $6.4 \%$ y otros $57.4 \%$. Mortalidad hospitalaria $77.8 \%$. Factores asociados a mortalidad: edad $(R M) 1.09(p=0.01)$ y la presión plateau RM $1.3(p=0.03)$; PDP RM $1.4(p=0.12)$, PM RM $0.79(p=0.07)$ y EVPP RM $1.5(p=0.7)$. Factores asociados a SOFA: SAPS $3(r)=0.53 p<0.0001$. SMR 1.06 IC 95\% 0.80-1.4 $\mathrm{p}=0.63$.

Conclusiones: La EVPP, PDP y el PM no tuvieron relación con la mortalidad ni SOFA en esta cohorte de pacientes.

Palabras clave: Estrategia ventilatoria de protección pulmonar, poder mecánico, presión distensión pulmonar, mortalidad, SOFA.

\section{ABSTRACT}

Introduction: Mechanical ventilation is the cornerstone of the treatment of patients with acute respiratory failure, however, it is not free of complications and eventual adverse events.

Objective: To describe the relationship between EVPP, PM and PDP with mortality and SOFA in adult patients with IMV attended at HGZ No. 11, IMSS, Piedras Negras, Coahuila.

Material and methods: Prospective, observational study, September 2019 to March 2020 of adult patients with invasive mechanical ventilation. The independent variable was $L P V, M P$ and DP. The dependent variables were mortality and SOFA.

Results: 63 patients were enrolled. Main characteristics: age 55 years female 44.4\%, location: ward 50.8\%, ER 17.5\% and ICU 31.8\%. Diagnoses: sepsis $20.6 \%$, severe TBI $7.8 \%$, stroke $7.8 \%$, CAP $6.4 \%$ and others $57.4 \%$. Hospital mortality $77.8 \%$. Factors associated with mortality: age OR $1.09(p=0.01)$ and plateau pressure OR $1.3(p=0.03)$; DP OR $1.4(p=0.12)$, MP OR $0.79(p=$ $0.07)$ and LPV OR $1.5(p=0.7)$. Factors associated with SOFA: SAPS $3(r)=$ $0.53 p<0.0001$. SMR $1.0695 \% \mathrm{Cl} 0.80-1.4 p=0.63$.

Conclusions: LPV, DP and MP were not related to mortality or SOFA in this cohort of patients.

* Hospital General de Zona No. 11, Unidad de Terapia Intensiva Piedras Negras Coahuila.

\section{Recibido: 08/10/2020. Aceptado: 09/02/2021}

Citar como: Rodríguez de Molina SJI, Castro MHH, Oyarvide ZAK, Flores OGA. Ventilación pulmonar protectora, poder mecánico y presión de distensión pulmonar relacionado con mortalidad y SOFA en ventilación mecánica invasiva. Med Crit. 2021;35(4):186-193. https://dx.doi.org/10.35366/101157

www.medigraphic.com/medicinacritica
Keywords: Lung protective ventilation, mechanical power, pulmonary distention pressure, mortality, SOFA.

\section{RESUMO}

Introdução: A ventilação mecânica é a pedra angular do tratamento de pacientes com insuficiência respiratória aguda, porém não está isenta de complicações e eventuais eventos adversos.

Objetivo: Descrever a relação entre EVPP, PM e PDP com mortalidade e SOFA em pacientes adultos com VMI tratados no HGZ 11 IMSS Piedras Negras Coahuila.

Material e métodos: Estudo prospectivo, observacional, de setembro de 2019 a março de 2020 em pacientes adultos com ventilação mecânica invasiva. $A$ variável independente foi EVPP, PM e PDP. As variáveis dependentes foram mortalidade e SOFA.

Resultados: 63 pacientes foram admitidos. Principais características: Idade 55 anos feminino 44.4\%, localização: enfermaria 50.8\%, emergência 17.5\% e UTI 31.8\%. Diagnósticos: Sepse 20.6\%, TCE grave 7.8\%, EVC 7.8\%, NAC 6.4\% e outros $57.4 \%$. Mortalidade hospitalar $77.8 \%$. Fatores associados à mortalidade: idade $(R M) 1.09(p=0.01)$ e pressão plateau RM $1.3(p=0.03) ;$ PDP RM 1.4 ( $p$ $=0.12)$, PM RM $0.79(p=0.07)$ e EVPP RM $1.5(p=0.7)$. Fatores associados ao SOFA: SAPS $3(r)=0.53 p<0.0001$. SMR 1.06 IC 95\% 0.80-1.4 $p=0.63$.

Conclusões: EVPP, PDP e PM não foram relacionados à mortalidade ou SOFA nesta coorte de pacientes.

Palavras-chave: Estratégia ventilatória de proteção pulmonar, potência mecânica, pressão de distensão pulmonar, mortalidade, SOFA.

\section{INTRODUCCIÓN}

La ventilación mecánica es la piedra angular del tratamiento de los pacientes con insuficiencia respiratoria aguda y es una de las terapéuticas que definen la medicina crítica como especialidad. ${ }^{1}$ El primer reporte formal de la ventilación mecánica fue realizado por el Dr. Bjorn Ibsen en Copenhague (1953), en pacientes con polio, reduciendo la mortalidad de 87 a $25 \% .^{2} \mathrm{La}$ epidemiología de la ventilación mecánica en situaciones de escasos recursos es poca y usualmente es una transpolación de la información de centros de alta especialidad. ${ }^{3}$

La ventilación mecánica tiene el objetivo principal de asistir en la eliminación de $\mathrm{CO}_{2}$ y/o favorecer el adecuado intercambio de oxígeno mientras el paciente está imposibilitado de hacerlo o por condiciones externas al sistema respiratorio que no le permiten hacerlo por voluntad propia. ${ }^{4,5}$

Los modos de ventilación mecánica han tenido diversas innovaciones tecnológicas al paso de los años; ${ }^{5} \sin$ embargo, hasta el momento no existe superioridad de 
un modo ventilatorio sobre otro. Existe una variedad de modos ventilatorios que utilizan combinaciones de los previamente dichos, además de la creación de modos inteligentes y automatizados. ${ }^{5}$

La ventilación mecánica no invasiva es una opción terapéutica para los pacientes que requieren asistencia respiratoria. ${ }^{1}$ La utilización de interfaces no invasivas: mascarilla nasal, oronasal, facial y casco cefálico han demostrado ser adecuadas en el perfil correcto de pacientes. ${ }^{6}$ La interfaz y su adecuado funcionamiento son parte fundamental para el éxito de este método de ventilación, además de tolerancia y cooperación del paciente. ${ }^{6}$

La falla respiratoria es la principal indicación de uso de ventilación mecánica; ésta puede ser: falla ventilatoria o falla para lograr una adecuada oxigenación..$^{7,8}$ La falla ventilatoria se caracteriza por un incremento en la presión arterial de $\mathrm{CO}_{2}\left(\mathrm{PaCO}_{2}\right)$, debido a hipoventilación alveolar o incremento del trabajo respiratorio. La falla para mantener una adecuada oxigenación se caracteriza por una anormalmente baja relación entre presión arterial de oxígeno y fracción inspirada de oxígeno $\left(\mathrm{PaO}_{2}: \mathrm{FiO}_{2}\right)$ y/o un requerimiento de presión positiva para mantener esta relación. 9, $10^{2}$

La insuficiencia respiratoria aguda se define como una alteración severa, súbita del intercambio gaseoso pulmonar que es caracterizada por la incapacidad de los pulmones de cumplir con las demandas metabólicas del cuerpo de transporte de oxígeno $\left(\mathrm{O}_{2}\right)$ y remoción de dióxido de carbono $\left(\mathrm{CO}_{2}\right) \cdot{ }^{11,12} \mathrm{El}$ diagnóstico se realiza mediante la medición de ph, $\mathrm{O}_{2}$ y $\mathrm{CO}_{2}$ por gasometría arterial.

Las complicaciones o eventos adversos asociados a la ventilación mecánica tienen una incidencia estimada de cuatro a siete eventos por cada 100 episodios de ventilación mecánica, o 10-15 eventos por cada 1,000 días en ventilación mecánica, éstas son: ${ }^{13}$

1. Neumonía o aspiración (36\%)

2. Edema pulmonar, derrame pleural o falla cardiaca $(28 \%)$

3. Atelectasia (14\%)

4. Síndrome de insuficiencia respiratoria aguda (9\%)

5. Sepsis o infección extrapulmonar (3.8\%)

6. Distensión abdominal o síndrome compartamental abdominal (3.5\%)

7. Evento neurológico agudo (2.9\%)

8. Embolismo pulmonar (1.2\%)

9. Neumotórax (1.2\%)

10. Tapón de moco $(0.3 \%)$

11. Mala higiene pulmonar $(0.3 \%)$

12. Neumonitis por radiación $(0.3 \%)$

13. Otras complicaciones (2.6\%)

Los eventos adversos en la ventilación mecánica están asociados con incremento en la mortalidad, duración de la ventilación mecánica, de la estancia en la Unidad de Terapia Intensiva y aumento en el uso de antibióticos. ${ }^{14}$

La neumonía asociada a la ventilación mecánica se define como aquella que se desarrolla 48 horas después de la intubación orotraqueal. ${ }^{15}$ Ésta tiene una prevalencia de $10-20 \%$ a nivel mundial. ${ }^{16} \mathrm{La}$ incidencia en países en desarrollo es de 16.7 a 73.4 por 1,000 días de ventilación mecánica invasiva. ${ }^{17}$

La lesión asociada a la ventilación mecánica (por su siglas en inglés VILI Ventilator Induced Lung Injury) es otra complicación, ${ }^{18}$ es resultado del barotrauma, volutrauma, atelectrauma, biotrauma y muy recientemente del ergotrauma. ${ }^{18} \mathrm{El}$ actual concepto de VILI es el resultado de una evolución de las estrategias de protección pulmonar, las cuales iniciaron con del Dr. Amato con la disminución de volúmenes tidales; posteriormente con la limitación de la presión meseta, más adelante con maniobras de reclutamiento alveolar y titulación de presión positiva al final de la espiración (PEEP) para intentar mantener el «pulmón abierto».

En la actualidad, ya se han establecido como medidas de protección pulmonar las siguientes maniobras: volumen corriente menor $8 \mathrm{~mL} / \mathrm{kg}$ de peso ideal, presión meseta o plateau menor $30 \mathrm{cmH}_{2} \mathrm{O}$; sin embargo, la tendencia apunta a considerar que la presión transpulmonar es mucho más importante que la presión localizada en la vía aérea, puesto que esta presión es la que realmente rodea al alveolo y es la que ayuda a disminuir la heterogeneidad de las áreas pulmonares mal ventiladas, lo que incrementa la capacidad pulmonar residual. ${ }^{18}$ Un subrogado para el cálculo de la presión transpulmonar es la presión de conducción o distensión pulmonar (presión plateau-PEEP), pues aunque no toma en cuenta las propiedades elásticas de la pared torácica, varios estudios experimentales han encontrado una correlación aceptable entre ésta y el estrés pulmonar. ${ }^{19}$

El concepto de poder mecánico fue tomado recientemente por el Dr. L Gattinoni y sus colaboradores: ellos presentaron y propusieron una descripción matemática del poder mecánico, en el cual la energía entregada por unidad de tiempo se describe como una entidad unificada y se explica con una ecuación. ${ }^{19}$

Poder mecánico $_{\mathrm{rs}}=(0.098) \cdot(\mathrm{FR} \cdot \Delta \mathrm{V}) \cdot\left(\mathrm{P}_{\text {pico }}-1 / 2 \cdot \Delta \mathrm{P}\right)$

El Dr. JJ Marinni propuso después una simplificación de esta fórmula sin tomar en cuenta los componentes dependientes del flujo y la resistencia. ${ }^{19}$

$$
\mathrm{PD}=(0.098) \cdot\left(\mathrm{P}_{\mathrm{PL}}-\mathrm{PEEP}\right) \cdot \mathrm{Vt} \cdot \mathrm{FR}
$$

En diversos estudios experimentales, bajo la premisa de la importancia de la cantidad de energía entregada al sistema respiratorio en la unidad de tiempo (Joule/min), se han dispuesto valores para determinar el umbral en el que se 
podría provocar VILI, calculando un poder mecánico no mayor a $12 \mathrm{~J} / \mathrm{min}$, esto en pacientes que se encuentran bajo ventilación mecánica invasiva en modalidades controladas.

Los indicadores de calidad de atención son la medida cuantitativa que se utiliza como guía para controlar y valorar la calidad de aspectos importantes de la práctica asistencial. ${ }^{20}$

Todos los indicadores tienen un comparador o estándar que se define como $^{20}$ el nivel deseado de cumplimiento para el indicador.

Los indicadores principales asociados al paciente en ventilación mecánica son: ${ }^{20}$

1. Profilaxis de úlceras por estrés en enfermos críticos que reciben nutrición enteral: la población en riesgo de hemorragia gastrointestinal ( $\mathrm{HGl}),{ }^{21}$ el estándar de cumplimiento de este indicador es de $80 \% .^{20}$

2. Estrategia de ventilación pulmonar protectora (EVPP) en el síndrome de insuficiencia respiratoria aguda (SIRA): la EVPP puede mejorar la supervivencia en los enfermos con SIRA, según criterios de Berlín. ${ }^{22,23}$ La población a la que se aplica es a todos los enfermos con SIRA en ventilación mecánica invasiva $>24$ horas. La EVPP se define como volumen tidal $<8$ $\mathrm{mL} / \mathrm{kg}$ de peso ideal y presión plateau $<30 \mathrm{cmH}_{2} \mathrm{O}$. El estándar de cumplimiento de este indicador es > $90 \%{ }^{20}$

3. Neumonía asociada a la ventilación mecánica: es habitualmente la infección nosocomial frecuente en la UTI, la importancia de su seguimiento deriva de su repercusión en la mortalidad y morbilidad que ocasiona, con prolongación de estancia en UTI. ${ }^{24} \mathrm{La}$ población a la que se aplica este indicador son todos los pacientes con ventilación mecánica invasiva de más de 24 horas. El estándar de cumplimiento es $<7$ episodios por 1,000 días de ventilación mecánica. ${ }^{20}$

4. Extubación accidental por maniobras: la retirada accidental de la cánula endotraqueal en enfermos en ventilación mecánica invasiva es un desenlace no deseable, puesto que se asocia con riesgo de reintubación y una mayor mortalidad. ${ }^{25}$ La población a la que se aplica es todos los enfermos que requieren ventilación mecánica invasiva a través de tubo endotraqueal.

El estándar de cumplimiento es $<3$ extubaciones accidentales por maniobras por 1,000 días de tubo endotraqueal. ${ }^{20}$

5. Reintubación: la reintubación de un enfermo por fallo de extubación se asocia a una mayor estancia hospitalaria y tasa de mortalidad. ${ }^{26}$ La extubación programada es aquella que se realiza de forma programada e intencionada por un profesional, la reintubación es la necesidad de colorar la cánula endotraqueal en las primeras 48 horas de extubación. La población aplicable son todas las extubaciones programadas, excluyendo extubación por limitación de esfuerzo te- rapéutico y reintubación por intervención quirúrgica. El estándar de cumplimiento es $<12 \%$. $^{20}$

En México, los estudios epidemiológicos han demostrado una tendencia a ser pacientes más jóvenes los que requieren ventilación mecánica invasiva $43-48$ años en promedio. ${ }^{27}$ La mortalidad de éstos es de $18-36 \% .{ }^{27}$

En nuestra comunidad, $55 \%$ de los pacientes atendidos en áreas críticas requieren ventilación mecánica invasiva. ${ }^{28}$ De esta población, $61.8 \%$ tenían un estado físico funcional, previo al ingreso terapia intensiva, su promedio de edad es 48.9 años. ${ }^{28}$ Los principales diagnósticos de estos pacientes son sepsis, estado postparocardiorrespiratorio y traumatismo craneoencefálico severo. ${ }^{28}$ La mortalidad hospitalaria reportada es $40.2 \%$. ${ }^{28}$

El impacto en la salud, social y económico en los pacientes que requieren ventilación mecánica invasiva es importante, aproximadamente $\$ 600-1,500$ dólares por día ${ }^{29}$ y las morbilidades posteriores al evento grave implicarán una carga para la familia y la sociedad.

El conocimiento del impacto y la implementación de estrategias de ventilación protectora pulmonar y las nuevas fórmulas de mecánica respiratoria nos darán la información para mejorar los protocolos de atención de este perfil de pacientes e influir en su evolución, disminuir complicaciones y posiblemente la mortalidad.

El objetivo principal de nuestro estudio fue describir la relación entre la estrategia de protección pulmonar, poder mecánico y la presión de distensión pulmonar con la mortalidad y el SOFA en pacientes adultos con ventilación mecánica invasiva atendidos en el Hospital General de Zona No. 11, IMSS, Piedras Negras, Coahuila.

Los objetivos secundarios fueron describir las características demográficas, el cumplimiento de aplicación de la estrategia de protección, las complicaciones asociadas a la ventilación mecánica invasiva, calcular los indicadores de calidad de atención más relevantes asociados a la ventilación mecánica invasiva y analizar la comparación entre la mortalidad real y la esperada por SAPS 3 de los pacientes con ventilación mecánica invasiva atendidos en HGZ No. 11, IMSS, Piedras Negras, Coahuila.

\section{MATERIAL Y MÉTODOS}

Se realizó una investigación con un diseño tipo prospectivo, observacional, longitudinal de septiembre 2019 a marzo 2020 de todos los pacientes adultos que requirieron ventilación mecánica invasiva atendidos en Hospital General de Zona No. 11, IMSS en Piedras Negras, Coahuila.

La variable independiente se definió como la estrategia de protección pulmonar, poder mecánico y la presión de distensión pulmonar.

Las variables dependientes fueron la mortalidad y el SOFA. 
La definición operacional de variables para este estudio y sus cálculos se encuentran especificados en el Anexo 1.

Los criterios de inclusión fueron pacientes adultos mayores de 18 años que estuvieron internados en el Hospital General de Zona No. 11, Piedras Negras, Coahuila y que requirieron ventilación mecánica invasiva.

Los criterios de exclusión fueron menores de 18 años y los criterios de eliminación fueron aquellos pacientes que posterior a firmar consentimiento de participación, declinaron esta decisión.

El análisis estadístico se realizó con Software StatCalc versión $1500.1 .3^{\odot}$ AcaStat software 2018 y epiinfo.com., las variables cuantitativas se describieron en medias y desviación estándar, las cualitativas en frecuencia y porcentaje, se realizará prueba de $\chi^{2}$ para encontrar una significancia $<0.05$ y análisis de regresión logística lineal y múltiple para determinar la significancia de asociación de las variables reportadas con la mortalidad y el SOFA. Se realizó correlación de Pearson para la estrategia de ventilación protectora con mortalidad, así como curvas ROC para evaluar la capacidad pronóstica de la presión de distensión pulmonar y el poder mecánico con la mortalidad hospitalaria.

La investigación no se realizó en población vulnerable, el riesgo de efectos secundarios fue nulo y se realizó con apego a las buenas prácticas clínicas, esto concordando con lo estipulado en la Ley General de Salud en Materia de Investigación en Salud, Declaración de Helsinki e International Council of Harmonisation.

La investigación fue aprobada por el Comité Local de Investigación en Salud 506 del Instituto Mexicano del Seguro Social (R-2019-506-010), además de contar con registro en clinicaltrials.gov con el numero NCT04193254.

Se solicitó carta de consentimiento informado a los pacientes incluidos en el estudio, se protegieron sus datos de identificación personal, siendo concordantes con la Ley de Protección de Información Personal.

El estudio no tuvo ningún financiamiento institucional o por empresas externas al mismo.

\section{RESULTADOS}

Las características principales de los pacientes fueron edad media $55 \pm 17.4(p<0.0001)$, femenino $44.4 \%$ ( $p$ $=0.3$ ), ubicación intrahospitalaria: piso $50.8 \%$, urgencias $17.5 \%$ y UTI $31.8 \%$ ( $<<0.0050)$. Los principales diagnósticos de esta cohorte se reflejan en la Tabla 1. La mortalidad hospitalaria fue de $77.8 \%$, dos pacientes fueron trasladados a otro hospital.

Las poblaciones atendidas en Urgencias, Piso y UTI tuvieron diferencias estadísticamente significativas en cuanto a la edad, horas de ventilación mecánica invasiva, SOFA y mortalidad hospitalaria (Tabla 2).
Se realizó análisis de regresión logística para evaluar los factores asociados a mortalidad, resultando estadísticamente significativos: la edad con razón de momios (RM) $1.09(p=0.01)$ y la presión plateau RM 1.33 ( $p$ $=0.03$ ); en cuanto a la variables a estudiar, la presión de distensión pulmonar RM $1.36(p=0.12)$, poder mecánico RM $0.79(p=0.07)$ y estrategia de ventilación protectora pulmonar (EVPP) RM $1.5(p=0.7)$ (Tabla 3).

Los factores asociados a SOFA fueron evaluados con análisis de regresión logística múltiple (Anexo 1) y correlación de Pearson, teniendo significancia estadística únicamente con el SAPS $3(r)=0.53 p<0.0001$ (Tabla 4).

Se realizaron curvas ROC (Receiver Operating Characteristic) para evaluar la capacidad de predecir la mortalidad de la presión de distensión pulmonar AUC 0.60 IC 95\% 043-0.78 (Figura 1), poder mecánico AUC

Tabla 1: Características principales de los pacientes con ventilación mecánica invasiva. $\mathrm{N}=63$.

\begin{tabular}{|c|c|c|}
\hline & $\mathrm{n}(\%)$ & $p$ \\
\hline Femenino & $28(44.4)$ & $<0.3700$ \\
\hline Edad (años) & $55.9 \pm 17.5$ & $<0.0001$ \\
\hline Índice de masa corporal & $30.5 \pm 8.1$ & $<0.0001$ \\
\hline Peso ideal o predicho & $60.5 \pm 11.9$ & $<0.0001$ \\
\hline Días en hospital & $12 \pm 12.5$ & $<0.0001$ \\
\hline Horas VMI & $169.6 \pm 215.6$ & $<0.0001$ \\
\hline SOFA & $9.1 \pm 4.5$ & $<0.0001$ \\
\hline SAPS 3 & $83.9 \pm 21.1$ & $<0.0001$ \\
\hline SAPS 3 mortalidad & $73.2 \pm 30.2$ & $<0.0001$ \\
\hline Mortalidad hospital & $49(77.8)$ & $<0.0001$ \\
\hline Traslados a otro hospital & $2(3.2)$ & $<0.0001$ \\
\hline \multicolumn{3}{|l|}{ Ubicación intrahospitalaria } \\
\hline Piso & $32(50.8)$ & $<0.0050$ \\
\hline Unidad de Terapia Intensiva & $20(31.8)$ & $<0.0050$ \\
\hline Quirófano & $11(17.4)$ & $<0.0050$ \\
\hline \multicolumn{3}{|l|}{ Parámetros respiratorios } \\
\hline Volumen tidal & $444.4 \pm 118.5$ & $<0.0001$ \\
\hline Presión plateau & $23.6 \pm 5.8$ & $<0.0001$ \\
\hline PEEP & $6.9 \pm 1.9$ & $<0.0001$ \\
\hline Frecuencia respiratoria & $19.1 \pm 5.1$ & $<0.0001$ \\
\hline DP & $16.5 \pm 5.4$ & $<0.0001$ \\
\hline Poder mecánico & $13.9 \pm 7.5$ & $<0.0001$ \\
\hline \multirow[t]{2}{*}{ Modo convencional VM } & $\mathrm{A} / \mathrm{C} P 27$ (42.9) & $<0.0001$ \\
\hline & $\mathrm{A} / \mathrm{C} \vee 15$ (23.9) & $<0.0001$ \\
\hline \multirow[t]{2}{*}{ Modo no convencional VM } & SIMV 4 (6.4) & $<0.0001$ \\
\hline & APRV 2 (3.1) & $<0.0001$ \\
\hline \multicolumn{3}{|l|}{ Principales diagnósticos } \\
\hline Sepsis & $13(20.6)$ & $<0.0001$ \\
\hline Traumatismo craneoencefálico severo & $5(7.8)$ & $<0.0001$ \\
\hline Evento vascular cerebral & $5(7.8)$ & $<0.0001$ \\
\hline $\begin{array}{l}\text { Neumonía adquirida en la } \\
\text { comunidad }\end{array}$ & $4(6.4)$ & $<0.0001$ \\
\hline Otros & $36(57.4)$ & $<0.0001$ \\
\hline
\end{tabular}

$\mathrm{VMI}=$ ventilación mecánica invasiva, SOFA = Sequential Organic Failure Assessment, SAPS3 = Simplified Acute Physiology Score 3, PEEP = Possitive End Expiratory Presssure, $v$ gr., presión al final de la espiración, DP = Driving Pressure, $\checkmark$ gr. presión de distensión pulmonar, $A / C P=$ asisto/controlado por presión, $A / C$ $\mathrm{V}=$ asisto/controlado por volumen, SIMV = Sincronized Intermitent Mechanical Ventilation, APRV = Airway Pressure Relieve Ventilation. 
Tabla 2: Características por sitio de ubicación intrahospitalaria de los pacientes con ventilación mecánica invasiva. N = 63 .

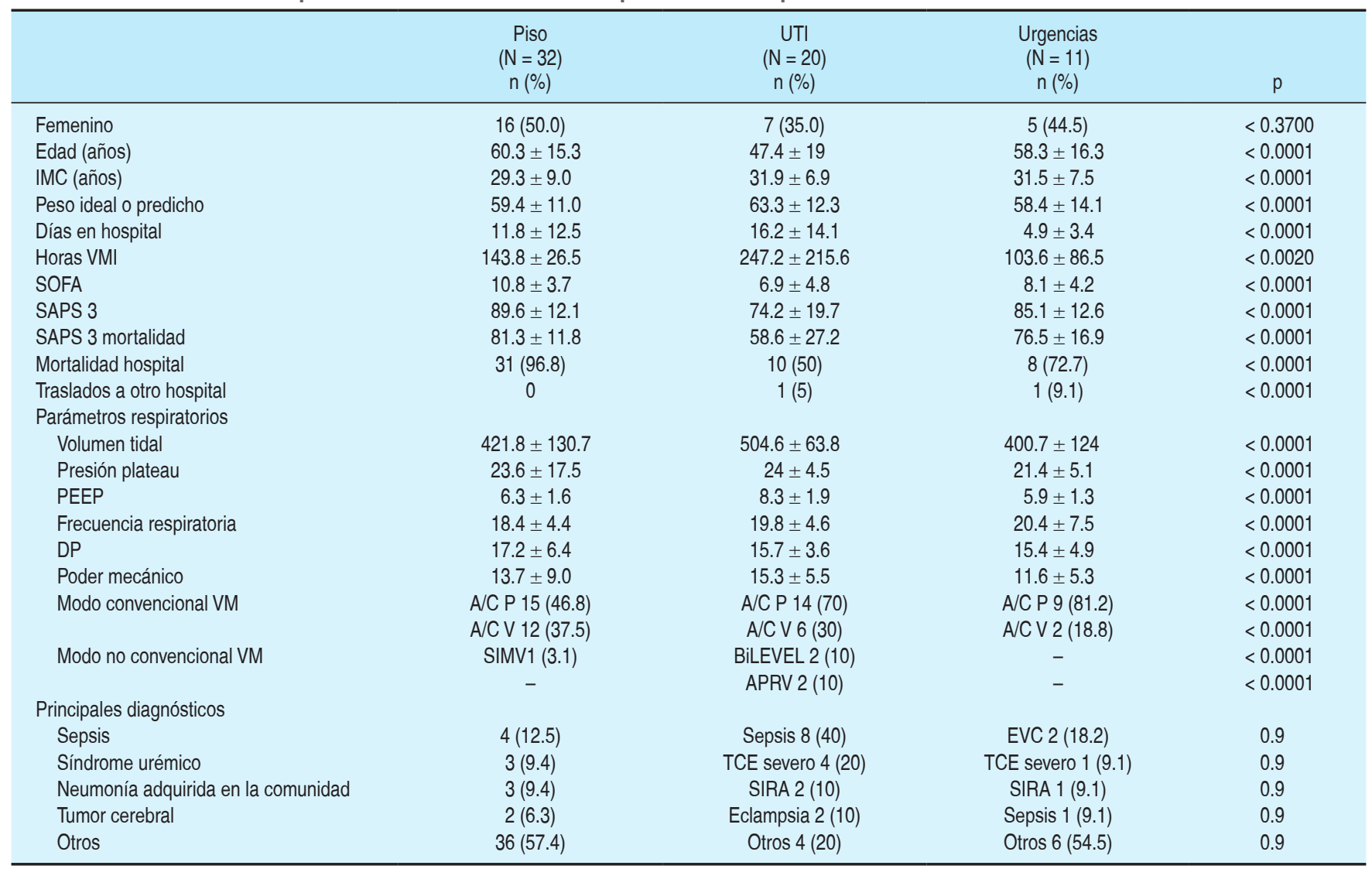

UTI = Unidad de Terapia Intensiva, IMC = índice de masa corporal, SOFA = Sequential Organic Failure Assessment, SAPS3 = Simplified Acute Physiology Score 3, PEEP $=$ Possitive End Expiratory Presssure, $\mathrm{v}$ gr., presión al final de la espiración. DP = Driving Pressure, $\mathrm{v}$ gr. presión de distensión pulmonar, $\mathrm{VMI}=$ ventilación mecánica invasiva, A/C P = asisto/controlado por presión, A/C V = asisto/controlado por volumen, SIMV = Sincronized Intermitent Mechanical Ventilation, BiLEVEL = Bilevel ventilation, APRV = Airway Pressure Relieve Ventilation, EVC = evento vascular cerebral, TCE = traumatismo craneoencefálico, SIRA = síndrome de insuficiencia respiratoria aguda.

0.41 IC 95\% 0.25-0.57 (Figura 2) y presión plateau AUC 0.53 IC 95\% 0.36-0.73 (Figura 3).

Los indicadores de calidad de atención en pacientes con ventilación mecánica fueron calculados según SEMICYUC (Sociedad Española de Medicina Intensiva Crítica y Unidades Coronarias) (Tabla 5).

La tasa de mortalidad estandarizada en la población de estudio fue SMR (standarized mortality ratio) 1.06 IC 95\% 0.80-1.4 p = 0.63 y dividido por áreas de atención fue: Piso SMR 1.19 IC 95\% 0.82-168 p=0.31, UTI SMR 0.85 IC 95\% 0.43-1.5 $p=0.64$ y Urgencias SMR 0.95 IC $95 \% 0.44-1.81 p=0.94$.

\section{DISCUSIÓN}

En nuestro estudio se reporta una mortalidad de $77.8 \%$ con un promedio de edad de $55.9 \pm 17.5(p=0.0001)$, ésta es mayor a la reportada en la literatura internacional $40.2 \%$ y nacional $28-45 \%,{ }^{19,28}$ con un promedio de edad de 48.9 años, siendo menor a lo reportado en México 60.8 años. ${ }^{18}$ Los principales diagnósticos fueron: sepsis, traumatismo craneoencefálico severo, evento vascular cerebral, neumonía adquirida en la comunidad; éstos difieren con los de la literatura internacional, los cuales reportan: neumonía o aspiración 36\%, edema pulmonar, derrame pleural o falla cardiaca $28 \%$, atelectasias $14 \%$, síndrome de insuficiencia respiratoria aguda $9 \%$, sepsis o infección extrapulmonar $3.8 \% .^{13}$

Un estudio previo realizado en población de la misma institución, los principales diagnósticos en este tipo de pacientes son sepsis, estado postparocardiorrespiratorio y traumatismo craneoencefálico severo. ${ }^{28}$

La relación entre la estrategia de protección pulmonar, poder mecánico y la presión de distensión pulmonar con la mortalidad y el SOFA no tuvo significancia estadística en nuestro estudio PM RM $0.79 p=0.07$, EPP $1.7 p=0.7$ y PDP RM $1.36 p=0.12$, aunque los valores obtenidos cumplieron en su mayoría las metas internacionales de protección pulmonar Vt $44.4 \pm 118.5$ $\mathrm{p}<0.0001$; Ppla $23.6 \pm 5.8 \mathrm{p}<0.0001$; DP $16.5 \pm 5.4$ $\mathrm{p}<0.0001$; PM $13.9 \pm 7.5 \mathrm{p}<0.0001$; SOFA media 9.1 $\pm 4.5 p<0.0001$, esto es diferente a lo reportado en la literatura nacional e internacional PM RM $1.64 p=0.02$ y RR $1.005 p<0.0005$, respectivamente. ${ }^{18,30,31}$ 
La diferencia entre la mortalidad real y la esperada, evaluada con la tasa de mortalidad estandarizada fue de 1.03 IC 95\% 0.8-1.4 p = 0.63, no teniendo significancia estadística en la población general y en el análisis de subgrupos.

Los resultados encontrados en este estudio difieren en los reportes nacionales e internacionales, esto puede deberse a la heterogeneidad de las poblaciones y a su localización intrahospitalaria. Este último rubro cobra vital importancia debido a que la mayoría de los estudios de ventilación mecánica invasiva se realizan en población en Terapia Intensiva.

Los indicadores de calidad de atención de los pacientes de VMI en este estudio se reportaron con incumplimiento de los mismos, esto impacta directamente a la evolución y mortalidad de los pacientes.

Las limitaciones de este estudio son el tamaño de muestra, la heterogeneidad derivada de la ubicación in-

Tabla 3: Análisis de regresión logística para las variables asociadas con la mortalidad hospitalaria en pacientes con ventilación mecánica invasiva.

\begin{tabular}{lccc}
\hline Variable & Coeficiente B & $\begin{array}{c}\text { Razón de } \\
\text { momios }\end{array}$ & $\mathrm{p}$ \\
\hline Horas de VMI & 0.0012 & 1.00 & 0.63 \\
Edad & 0.0860 & 1.09 & 0.01 \\
Sexo & 2.2076 & 9.09 & 0.11 \\
Peso & -0.0732 & 0.92 & 0.74 \\
Talla & 1.8950 & 6.70 & 0.93 \\
IMC & 0.2588 & 1.30 & 0.66 \\
Vt promedio & -0.0121 & 0.98 & 0.05 \\
Pplat & 0.2916 & 1.33 & 0.03 \\
PEEP promedio & -0.0447 & 0.96 & 0.87 \\
FR promedio & -0.1950 & 0.82 & 0.11 \\
PDP & 0.3089 & 1.36 & 0.12 \\
PM & -0.2348 & 0.79 & 0.07 \\
EVPP & 0.3913 & 1.50 & 0.70 \\
NAV & -0.7067 & 0.49 & 0.45 \\
SOFA promedio & 0.1073 & 1.11 & 0.42 \\
SAPS3 & 0.0711 & 1.07 & 0.06 \\
\hline
\end{tabular}

$\mathrm{VMI}=$ ventilación mecánica invasiva, IMC = índice de masa corporal, $\mathrm{Vt}=$ volumen tidal, Pplat $=$ presión plateau, $\mathrm{PEEP}=$ Positive End Expiratory Pressure, $\mathrm{FR}=$ frecuencia respiratoria, $\mathrm{PDP}=$ Presión de distensión pulmonar, $\mathrm{PM}=$ poder mecánico, EVPP = estrategia de ventilación pulmonar protectora, NAV = neumonía asociada a la ventilación mecánica, SOFA = Sequential Organ Failure Assessment SAPS3 $=$ Simplified Acute Physiology Score 3.

Tabla 4: Correlación de Pearson para las variables asociadas con SOFA en pacientes con ventilación mecánica invasiva.

\begin{tabular}{lcc}
\hline Variable & $\begin{array}{c}\text { Coeficiente de } \\
\text { correlación }(r)\end{array}$ & $p$ \\
\hline Presión de distensión pulmonar & 0.13 & 0.30 \\
Poder mecánico & 0.17 & 0.18 \\
Simplified Acute Physiology Score 3 & 0.53 & $<0.0001$ \\
\hline
\end{tabular}

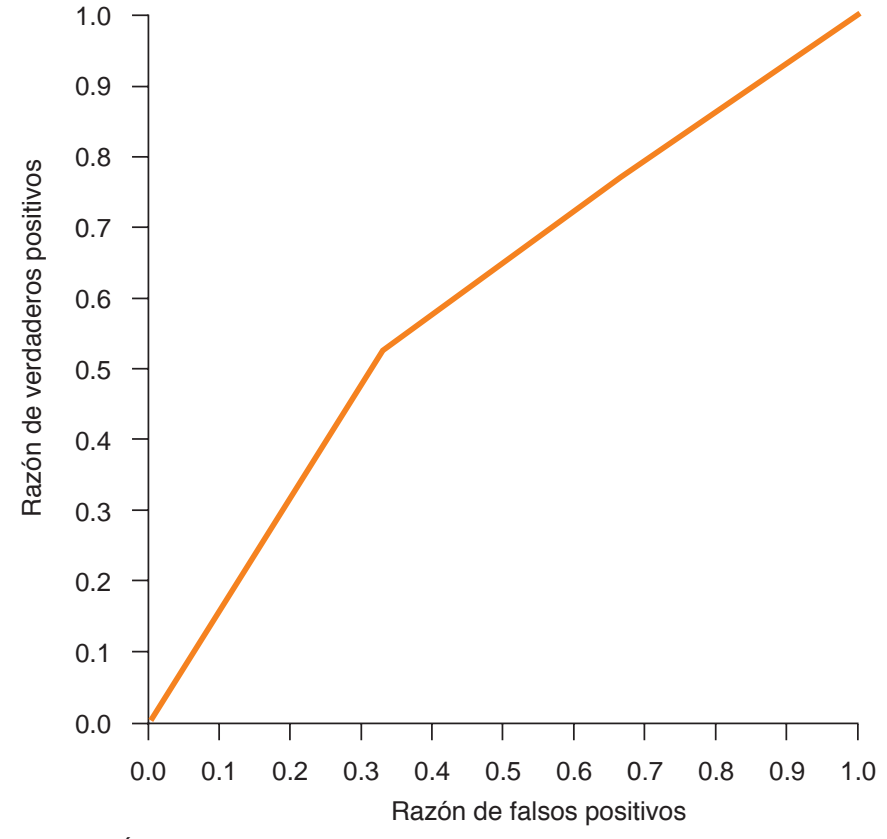

Área bajo la curva COR $=0.6020408$ (0.4243201-0.7797614)

Figura 1: Curva COR PDP y mortalidad.

$\mathrm{COR}=$ característica operativa del receptor, $\mathrm{PDP}=$ presión de distensión pulmonar.

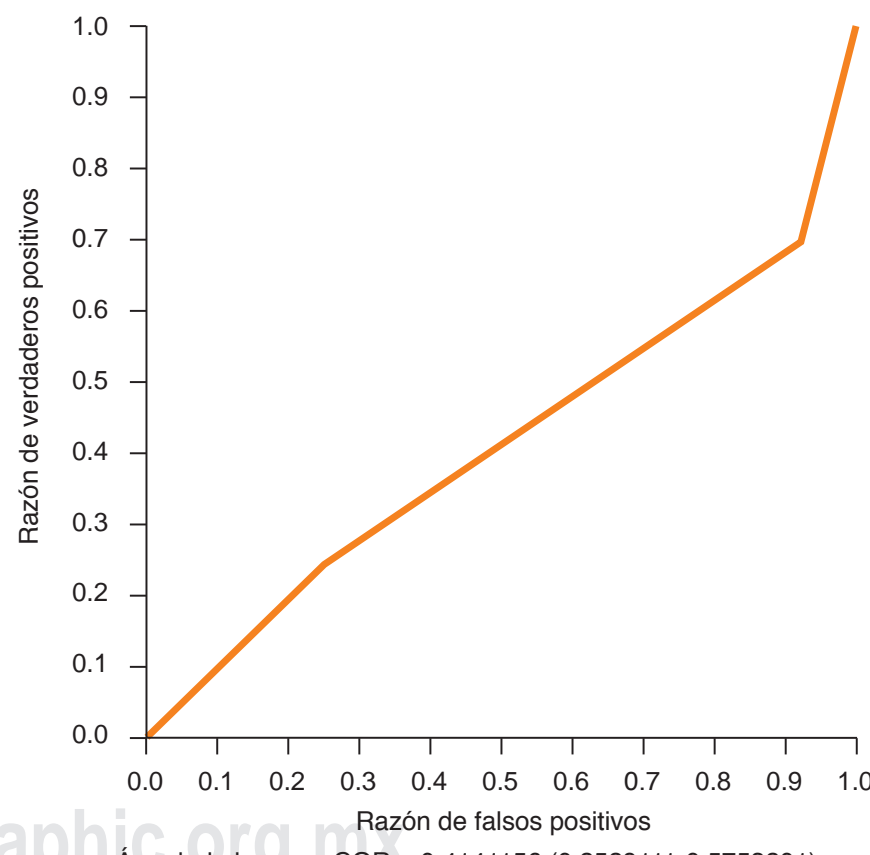

Área bajo la curva COR $=0.4141156(0.2529111-0.5753201)$

Figura 2: Curva COR PM y mortalidad.

$\mathrm{COR}=$ característica operativa del receptor, $\mathrm{PM}=$ poder mecánico.

trahospitalaria y las diferencias de atención médica y de enfermería dependientes de las mismas. Las fortalezas provienen de la representación de datos en «vida real» de los pacientes con VMI que se atienden dentro y fuera de la UTI, situación común en los hospitales del servicio de salud pública de México. 


\section{CONCLUSIONES}

La EVPP, PDP y el PM no tuvieron ninguna relación con la mortalidad ni con el SOFA en esta cohorte de pacientes, la presión plateau sí tuvo una relación estadísticamente significativa, aunque un rendimiento malo con AUC 0.54. El tamaño de la muestra y la variabilidad de las poblaciones y el manejo ventilatorio aplicado pueden tener un impacto en nuestros resultados; sin embargo, proporcionamos datos de las características clínicas y la evolución en «vida real» de los pacientes atendidos en hospitales del servicio de salud público en México donde, derivado de la escasez de recursos y espacios, no todos los pacientes con ventilación mecánica invasiva son tratados en UTI. La falta de cumplimiento de los indicadores de calidad de atención en pacientes con VMI demostrada en esta cohorte tiene un impacto

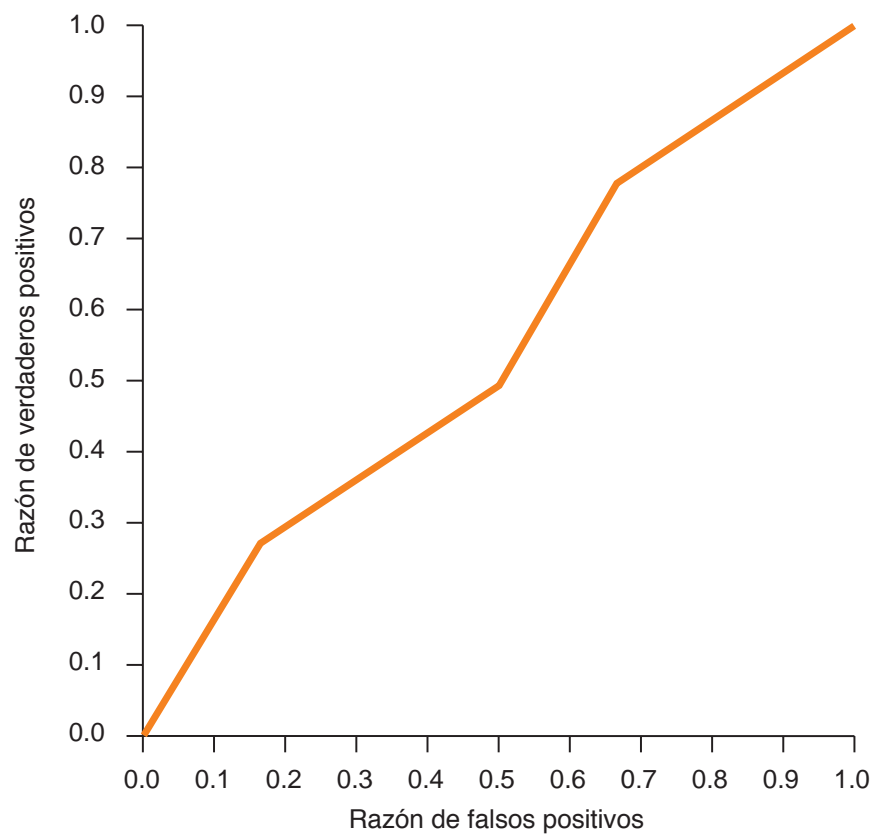

Área bajo la curva COR $=0.5493197(0.3661229-0.7325164)$

Figura 3: Curva COR Pplat y mortalidad.

$\mathrm{COR}=$ característica operativa del receptor, Pplat = presión plateau.

Tabla 5: Indicadores de calidad de atención en pacientes con ventilación mecánica invasiva.

\begin{tabular}{lcc}
\hline Indicador & Estándar & Valor obtenido \\
\hline Profilaxis UG, \% & 80 & 82.5 \\
Profilaxis TVP, \% & 90 & 77.7 \\
Cumplimiento EVPP & $>90$ & 50.8 \\
NAV (días de VMI) & $<7 / 1,000$ & $42.6 / 1,000$ \\
Extubación accidental (días de VMI) & $<3 / 1,000$ & $38.1 / 1,000$ \\
Reintubación, \% & $<12$ & 35.3 \\
\hline
\end{tabular}

UG = úlceras gástricas, $T V P=$ trombosis venosa profunda, $E V P P=$ estrategia ventilatoria pulmonar protectora, NAV = neumonía asociada a la ventilación mecánica invasiva, $\mathrm{VMI}$ = ventilación mecánica invasiva. en la evolución y la mortalidad y nos compromete a mejorar los protocolos de atención y asistencia de estos pacientes en nuestra institución.

\section{REFERENCIAS}

1. Esteban A, Frutos-Vivar F, Muriel A, Ferguson ND, Peñuelas $\mathrm{O}$, et al. Evolution of mortality over time in patients receiving mechanical ventilation. Am J Respir Crit Care Med. 2013;188(2):220-230. doi: 10.1164/rccm.201212-21690C.

2. Mandelzweig K, Leligdowicz A, Murthy S, Lalitha R, Fowler RA, Adhikari NKJ. Noninvasive ventilation in \&\& children and adults in low- and low-middle income countries: a systematic review and meta-analysis. J Crit Care. 2018;47:310-319. doi: 10.1097/ MCC.0000000000000568.

3. DynaMed [Internet]. Ipswich (MA): EBSCO Information Services. 1995-. Record No. 253048, Mechanical ventilation; [updated 2018 Nov 07, cited place cited date here]; [about 36 screens]. Available from: http://search.ebscohost.com/ login. asp $x$ ?direct=true $\& \mathrm{db}=\mathrm{dnh} \& \mathrm{AN}=253048 \&$ site $=$ dynamedlive\&scope $=$ site

4. ACCP Critical Care Medicine Board Review. 21st ed. 2012.

5. Huan YT, Singh J. Basic modes of mechanical ventilation. In: Papadakos P, Lachmann B, eds. Mechanical ventilation: clinical applications and pathophysiology. Philadelphia, PA: Saunders Elsevier; 2007:247-255.

6. Prescott HC, Brower RG, Cooke CR, Phillips G, O'Brien JM, National Institutes of Health Acute Respiratory Distress Syndrome Investigators. Factors associated with elevated plateau pressure in patients with acute lung injury receiving lower tidal volume ventilation. Crit Care Med. 2013;41(3):756-764.

7. Walls R. The decision to intubate. In: Walls R, Murphy M, eds. Manual of emergency airway management. 4th ed. Philadelphia, PA: Lippincott, Williams, and Wilkins; 2012, 1-7.

8. Tobin, MJ. Advances in mechanical ventilation. $N$ Engl J Med. 2001;344(26):1986-1996. doi: 10.1056/NEJM200106283442606.

9. Roussos C, Koutsoukou A. Respiratory failure. Eur Respir J. 2003:47:3s-14s.

10. Lascarrou JB, Boisrame-Helms J, Bailly A, Le Thuaut A, Kamel $\mathrm{T}$, Mercier $\mathrm{E}$, et al. Video laryngoscopy vs direct laryngoscopy on successful first-pass orotracheal intubation among ICU patients: a randomized clinical trial. JAMA. 2017;317(5):483-493. doi: 10.1001/jama.2016.20603.

11. Vincent JL, Abraham E, Moore FA, Kochanek P, Fink MP. Textbook of critical care. 7ed. Chapter 9 Acute Respiratory Failure. 7ed. Philadelphia: Elsevier. 2017, 33-37.

12. Wunsch H, Linde-Zwirble WT, Angus DC, Hartman ME, Milbrandt $\mathrm{EB}, \mathrm{Kahn}$ JM. The epidemiology of mechanical ventilation use in the United States. Crit Care Med. 2010;38(10). doi: 10.1097/ CCM.0b013e3181ef4460.

13. Centers for Disease Control and Prevention (CDC). VentilationAssociated Event. Report 2019. https://www.cdc.gov/nhsn/pdfs/ pscmanual/10-vae_final.pdf

14. Chalmers JD. Healthcare-associated pneumonia does not accurately identify potentially resistant pathogens: a systematic review and meta-analysis. Clin Infect Dis. 2014;58(3):330-339. doi: $10.1093 /$ cid/cit734.

15. Kollef MH, Chastre J, Fagon JY, Francois B, Niederman MS, Rello J, et al. Global prospective epidemiologic and surveillance study of ventilator-associated pneumonia due to Pseudomonas aeruginosa. Crit Care Med. 2014;42(10):2178-2187. doi: 10.1097/CCM.0000000000000510.

16. Rasslan O, Seliem ZS, Ghazi IA, El Sabour MA, El Kholy AA, Sadeq FM, et al. Device-associated infection rates in adult and pediatric intensive care units of hospitals in Egypt. International Nosocomial Infection Control Consortium (INICC) findings. $J$ Infect Public Health. 2012;5(6):394-402. doi: 10.1016/j. jiph.2012.07.002.

17. Indicadores de calidad del enfermo crítico actualización 2017. Sociedad española de medicina intensiva crítica y unidades coronarias (SEMICYUC). Disponible en: https://semicyuc. 
org/wp-content/uploads/2007/04/indicadoresdecalidad2017_ semicyuc_spa-1.pdf

18. Fuentes GAJ, Monares ZE, Aguirre SJS, Franco GJ. El poder mecánico permite predecir mortalidad en pacientes en ventilación mecánica invasiva prolongada. Med Crit. 2019;33(1):10-14.

19. Gómez RJI, Monares ZE, González CBG, Camarena AG, Aguirre SJS, Franco GJ. Determinación del poder mecánico en pacientes en ventilación mecánica invasiva en modalidad espontánea. Med Crit. 2018;32(1):20-26.

20. Buendegens L, BueKoch A, Tacke F. Prevention of stress-related ulcer bleeding at the intensive care unit: risks and benefits of stress ulcer prophylaxis. World L Crit Care Med. 2016;5(1):57-64.

21. Krag M, Perner A, Wetterslev J, Wise MP, Hylander Moller M. Stress ulcer prophylaxis versus placebo or no prophylaxis in critically ill patients. A systematic review of randomised clinical trials with meta-analysis and trial sequential analysis. Intensive Care Med. 2014;40(1):11-12.

22. ARDS Definition Task Force, Ranieri VM, Rubenfeld GD, Thompson BT, Ferguson ND, Caldwell E, et al. Acute respiratory distress syndrome: the Berlin definition. JAMA. 2012;307(23):2526-2533.

23. Álvarez LF, Sánchez GM, Lorente L, Gordo F, Añón JM, Álvarez $\mathrm{J}$, et al. Guidelines for the prevention of ventilator-associated pneumonia and their implementation. The Spanish "Zero-VAP" bundle. Med Intensiva. 2014;38(4):226-236.

24. Pham JC, Williams TL, Sparnon EM, Cillie TK, Scharen HF, Marella WM. Ventilator-related adverse events: a taxonomy and findings from 3 incident reporting systems. Respir Care. 2016;61(5):621-631.

25. Frutos-Vivar F, Esteban A, Apezteguia C, González M, Arabi Y, Restrepo MI, et al. Outcome of reintubated patients after scheduled extubation. J Crit Care. 2011;26(5):502-509.
26. http://www.cenetec.salud.gob.mx/descargas/gpc/ CatalogoMaestro/IMSS-687-PACIENTE_OBESO-DOSIS_DE_ FARMACOS/IMSS-687-13-GRR-PACIENTTE_OBESO-DOSIS_ FARMACOS.pdf

27. Marínae MC, Elizalde J, Villagómez A, Cerón U, Poblano M, Palma-Lara I, et al. ¿Se han producido cambios en la aplicación de la ventilación mecánica en relación con la evidencia científica? Estudio multicéntrico en México Med Intensiva. 2019. Disponible en: https://doi.org/10.1016/j.medin.2019.03.010

28. Rodriguez de Molina JI. Mechanical ventilation in low-resource setting: a descriptive analysis of two year experience at Piedras Negras, Coahuila, Mexico EC pulmonology and respiratory medicine. 8.8 2019, 615-622.

29. Klompas M. Potential strategies to prevent ventilator-associated events. Am J Respir Crit Care Med. 2015;192(12):1420-1430. doi: 10.1164/rccm.201506-1161Cl.

30. García-Prieto E, Amado-Rodríguez L, Albaiceta GM. Monitorización de la mecánica respiratoria en el paciente ventilado. Med Intensiva. 2014;38(1):49-55. Disponible en: http:// dx.doi.org/10.1016/j.medin.2013.09.003

31. Guérin C, Papazian L, Reignier J, Ayzac L, Loundou A, Forel JM. Effect of driving pressure on mortality in ARDS patients during lung protective mechanical ventilation in two randomized controlled trials. Critical Care. 2016;20:384. doi: 10.1186/s13054016-1556-2.

\author{
Correspondencia: \\ Dr. José Iván Rodríguez de Molina Serrano \\ E-mail: jirdems@gmail.com \\ https://orcid.org/0000-0001-9006-9586.
}

Anexo 1: Análisis de regresión logística múltiple para las variables asociadas con SOFA en pacientes con ventilación mecánica invasiva.

\begin{tabular}{cccc}
\hline Variable & Coeficiente B & Coeficiente estandarizado & $p$ \\
\hline Edad & 0.08505 & 0.3321 & 0.99 \\
Sexo & -0.75595 & -0.8395 & 0.07 \\
IMC & -0.03897 & -0.06999 & 0.60 \\
Vt promedio & 0.00833 & 0.22043 & 0.51 \\
Pplat & 0.11780 & 0.09961 & 1.0 \\
PEEP promedio & 0.08835 & 0.05615 & 1.0 \\
FR promedio & -0.08181 & -0.9002 & 0.73 \\
PDP & 0.02945 & 0.087 & 1.0 \\
PM & 0.07716 & 0.12023 & 0.80 \\
EVPP & -2.2529 & -0.25099 & 0.12 \\
SAPS3 & 0.15133 & 0.53 & $<0.0000$ \\
\hline
\end{tabular}

$\mathrm{VMI}=$ ventilación mecánica invasiva, IMC = índice de masa corporal, $\mathrm{Vt}=$ volumen tidal, Pplat $=$ presión plateau, $\mathrm{PEEP}=$ Positive End Expiratory Pressure, $\mathrm{FR}=$ frecuencia respiratoria, $\mathrm{PDP}=$ Presión de distensión pulmonar, $\mathrm{PM}=$ poder mecánico, $\mathrm{EVPP}=$ estrategia de ventilación pulmonar protectora, NAV = neumonía asociada a la ventilación mecánica, SOFA = Sequential Organ Failure Assessment, SAPS3 = Simplified Acute Physiology Score 3. 